\title{
FREDHOLM THEORY OF TOEPLITZ OPERATORS ON STANDARD WEIGHTED FOCK SPACES
}

\author{
Aamena Al-Qabani and Jani A. Virtanen \\ University of Reading, Department of Mathematics \\ Whiteknights, Reading RG6 6AX, England; rn030601@reading.ac.uk \\ University of Reading, Department of Mathematics \\ Whiteknights, Reading RG6 6AX, England; j.a.virtanen@reading.ac.uk
}

\begin{abstract}
We study the Fredholm properties of Toeplitz operators with bounded symbols of vanishing mean oscillation in the complex plane. In particular, we prove that the Toeplitz operator with such a symbol is Fredholm on a standard weighted Fock space if and only if the Berezin transform of the symbol is bounded away from zero outside a sufficiently large disk in the complex plane. We also show that the Fredholm index of the Toeplitz operator can be computed via the winding of the symbol along a sufficiently large circle. We finish by considering Toeplitz operators with matrix-valued symbols.
\end{abstract}

\section{Introduction}

Toeplitz operators $T_{f}$ form one of the most important classes of nonselfadjoint operators and it is remarkable that so much can be said about their spectral properties. In Hardy spaces $H^{p}$ of the unit circle, each Toeplitz operator has a simple representation as an infinite matrix constant along the parallels to its main diagonal; in particular, the $(i, j)$-entry of $T_{a}$ is given by the Fourier coeffient $a_{i-j}$ of the measurable function $a$. In general, such representations do not exist for Toeplitz operators acting on other function spaces, such as Bergman spaces $A^{p}$ of the unit disk $\mathbf{D}$ and Fock spaces $F_{\alpha}^{p}$ of the entire complex plane $\mathbf{C}$.

Our focus here is on the Fredholm properties of Toeplitz operators $T_{f}$ on $F_{\alpha}^{p}$, including the computation of the index formula and descriptions of the essential spectrum in terms (of the Berezin transform $\tilde{f}$ ) of the bounded symbol $f$ that generates $T_{f}$. In particular, we generalize the results of Berger and Coburn [3] and Stroethoff [17] on the essential spectra of Toeplitz operators on the unweighted Fock space $F^{2}$ with bounded symbols $f$ for which the corresponding Hankel operator $H_{f}$ is compact; that is, we show that for $f \in L^{\infty} \cap V M O^{1}$

$$
\sigma_{\mathrm{ess}}\left(T_{f}\right)=\bigcap_{R>0} \operatorname{cl} \tilde{f}(\mathbf{C} \backslash R \mathbf{D}),
$$

where cl $X$ denotes the closure of the set $X$ and $V M O^{1}$ stands for the functions of vanishing mean oscillation defined below. We also observe that

$$
\left\{f \in L^{\infty}: H_{f} \text { is compact }\right\}=L^{\infty} \cap V M O^{1},
$$

which was proved in $[3,17,19]$ for Hankel operators on the Fock-Hilbert spaces $F_{\alpha}^{2}$.

https://doi.org/10.5186/aasfm.2018.4344

2010 Mathematics Subject Classification: Primary 47B35; Secondary 30H20.

Key words: Toeplitz operators, Hankel operators, Fock spaces, Fredholm properties, essential spectrum, compactness, block Toeplitz operators, matrix-valued symbols.

J. Virtanen was supported in part by Engineering and Physical Sciences Research Council grant EP/M024784/1. 
The importance of Fredholm theory, as a fundamental part of spectral theory, stems from the role it plays in (global) analysis, many other parts of mathematics and various applications. The study of the Fredholm properties of Toeplitz operators dates back to the 1950s when Hartman and Wintner showed that the essential range of a bounded symbol $a: \partial \mathbf{D} \rightarrow \mathbf{C}$ is contained in the essential spectrum of $T_{a}$ acting on the Hardy-Hilbert space $H^{2}$. Since then there has been a steady increase in the study of spectral properties of Toeplitz operators on Hardy spaces and Bergman spaces, and in particular there is a good understanding of their spectral properties; for example, geometric descriptions of the spectra and essential spectra of Toeplitz operators have been obtained for several classes of symbols, such as continuous, piecewise continuous, (locally) sectorial, (semi) almost periodic, and Fisher-Hartwig symbols.

The situation regarding Toeplitz operators on Fock spaces is very different. While in the last ten years a considerable effort has been devoted to the study of boundedness, compactness and Schatten class properties of Toeplitz and Hankel operators on Fock spaces, see $[10,11,12,13,14,15,16,19]$, much less attention has been paid to the other important properties of these operators in the setting of Fock spaces. In fact, there are only two articles, both published more than two decades ago, which deal with the Fredholm properties of Toeplitz operators acting on the standard Fock space $F^{2}$, see $[3,17]$, and one recent work in the other (weighted) Fock spaces $F_{\alpha}^{p}$; see [7]. The understanding of the Fredholm properties of Toeplitz operators in Hardy and Bergman spaces is significantly superior and we only refer to [5] for Hardy spaces and [18] for the Bergman space, which are the two leading monographs on the subject of Toeplitz operators and their Fredholm properties in these two function spaces.

Our goal is to improve the level of understanding of Fredholm theory of Toeplitz operators, review some known results that may be useful for further advances, and list open problems. We hope this will stimulate further research into Fredholm theory in Fock spaces and lead to discoveries of new symbol classes for which meaningful criteria for Fredholmness can be established as in Hardy and Bergman spaces. Our main results and their proofs are given in Section 4.

\section{Preliminaries}

For $\alpha>0$ and $1 \leq p<\infty$, we write $L_{\alpha}^{p}=L^{p}\left(\mathbf{C}, d \mu_{p \alpha / 2}\right)$ for the space of Lebesgue measurable functions with respect to the Gaussian measure

$$
d \mu_{\alpha}(z)=\frac{\alpha}{\pi} e^{-\alpha|z|^{2}} d A(z)
$$

where $d A$ is the standard area measure on the complex plane. Obviously, we have $f \in L_{\alpha}^{p}$ if and only if the function $f(z) e^{-\alpha|z|^{2} / 2}$ is in $L^{p}(\mathbf{C}, d A)$. Also observe that for $f \in L_{\alpha}^{p}$,

$$
\|f\|_{p, \alpha}^{p}=\frac{p \alpha}{2 \pi} \int_{\mathbf{C}}|f(z)|^{p} d \mu_{p \alpha / 2}(z)=\frac{p \alpha}{2 \pi} \int_{\mathbf{C}}|f(z)|^{p} e^{-\frac{p \alpha}{2}|z|^{2}} d A(z) .
$$

The standard weighted Fock space $F_{\alpha}^{p}$ is defined by

$$
F_{\alpha}^{p}=\left\{f \in L_{\alpha}^{p}: f \text { is entire }\right\} .
$$

We note that each Fock space $F_{\alpha}^{p}$ is a closed subspace of $L_{\alpha}^{p}$ and hence itself a Banach space. If $0<p<q<\infty$, then $F_{\alpha}^{p} \subset F_{\alpha}^{q}$ and

$$
\|f\|_{q, \alpha} \leq(q / p)^{1 / q}\|f\|_{p, \alpha}
$$

for $f \in F_{\alpha}^{p}$ (see Theorem 2.10 of [19]). 
We write $F^{p}$ for the standard (unweighted) Fock spaces $F_{1 / 2}^{p}$. We say that $f \in L_{\alpha}^{\infty}$ if $f$ is Lebesgue measurable on $\mathbf{C}$ and

$$
\|f\|_{\infty, \alpha}=\operatorname{essiup}_{z \in \mathbf{C}}|f(z)| e^{-\frac{\alpha}{2}|z|^{2}}<\infty .
$$

The Fock space $F_{\alpha}^{2}$ is a Hilbert space with inner product given by

$$
(f, g)_{\alpha}=\int_{\mathbf{C}} f(z) \overline{g(z)} d \mu_{\alpha}(z)
$$

By the Riesz representation theorem, for $w \in \mathbf{C}$, there is a unique function $K_{w}$ in $F_{\alpha}^{2}$ such that

$$
f(w)=\left(f, K_{w}\right) \text { for all } f \in F_{\alpha}^{2} .
$$

The function $K_{\alpha}(z, w)=K_{w}(z)$ is called the reproducing kernel of $F_{\alpha}^{2}$. For $z \in \mathbf{C}$, we define

$$
k_{z}(w)=k_{z, \alpha}(w)=k_{\alpha}(w, z)=\frac{K(w, z)}{\sqrt{K(z, z)}}
$$

which is called the normalized reproducing kernel function at $z$. It is well known that $\left\|k_{z}\right\|_{p, \alpha}=1$ for all $z \in \mathbf{C}$ and $1 \leq p<\infty$. Note that the reproducing kernel $K_{\alpha}(z, w)$ of $F_{\alpha}^{2}$ has the explicit form

$$
K_{\alpha}(z, w)=e^{\alpha z \bar{w}} \quad \text { and so } \quad k_{z}(w)=e^{\alpha \bar{z} w-\frac{\alpha}{2}|z|^{2}} .
$$

We denote by $P_{\alpha}$ the orthogonal projection of $L_{\alpha}^{2}$ onto $F_{\alpha}^{2}$. The projection $P_{\alpha}$ can be expressed as the following integral operator

$$
\operatorname{Pf}(z)=\int_{\mathbf{C}} K_{\alpha}(z, w) f(w) d \mu_{\alpha}(w)
$$

for $z \in \mathbf{C}$. For any $\alpha>0$ and $1 \leq p \leq \infty$, the integral operator $P_{\alpha}$ is a bounded projection of $L_{\alpha}^{p}$ onto $F_{\alpha}^{p}$; the boundedness in the endpoint cases is in stark contrast with the situation in Hardy and Bergman spaces.

For $f \in L^{\infty}, \alpha>0$, and $1<p<\infty$, we define the Toeplitz operator $T_{a}: F_{\alpha}^{p} \rightarrow F_{\alpha}^{p}$ and the Hankel operator $H_{a}: F_{\alpha}^{p} \rightarrow L_{\alpha}^{p}$ by

$$
T_{a} f=P(a f) \quad \text { and } \quad H_{a} f=(I-P)(a f)
$$

for $f \in F_{\alpha}^{p}$. Clearly, $T_{a}$ and $H_{a}$ are bounded linear operators, and

$$
T_{a} f(z)=\int_{\mathbf{C}} K_{w}(z) a(w) f(w) d \mu_{\alpha}(w)
$$

for $z \in \mathbf{C}$. We only deal with Toeplitz and Hankel operators with bounded symbols and only consider them on the Fock spaces $F_{\alpha}^{p}$ with $1<p<\infty$. Some references are given for unbounded symbols and generalized Fock spaces in the next section.

In order to derive useful formulas for Toeplitz and Hankel operators at the kernel function, we define three analytic self-maps of the complex plane. For $\lambda \in \mathbf{C}$, set

$$
t_{\lambda}(z)=z+\lambda, \quad \tau_{\lambda}(z)=z-\lambda, \quad \varphi_{\lambda}(z)=\lambda-z .
$$


The mapping $t_{\lambda}$ is called the translation by $\lambda$, and clearly $\tau_{\lambda}=t_{-\lambda}=t_{\lambda}^{-1}$ and $\varphi_{\lambda}^{-1}=\varphi_{\lambda}$. The following useful formulas follow from a change of variables:

$$
\begin{aligned}
\int_{\mathbf{C}}\left|f \circ t_{\lambda}(w)\right|^{p} d \mu_{p \alpha / 2}(w) & =\frac{p \alpha}{2 \pi} \int_{\mathbf{C}}|f(w+\lambda)|^{p} e^{-\frac{p \alpha}{2}|w|^{2}} d A(w) \\
& =\frac{p \alpha}{2 \pi} \int_{\mathbf{C}}|f(z)|^{p} e^{-\frac{p \alpha}{2}|z-\lambda|^{2}} d A(z) \\
& =\frac{1}{\left\|K_{\lambda}\right\|_{p, \alpha}^{p}} \int_{\mathbf{C}}|f(z)|^{p}\left|K_{\lambda}(z)\right|^{p} d \mu_{p \alpha / 2}(z) \\
& =\int_{\mathbf{C}}|f(z)|^{p}\left|k_{\alpha}(z, \lambda)\right|^{p} d \mu_{p \alpha / 2}(z) .
\end{aligned}
$$

For $\lambda \in \mathbf{C}$, using (11), we get

$$
K_{z}(w)=K_{z}(\lambda) K_{z}(w-\lambda)=\overline{K_{\lambda}(z)} K_{z-\lambda}(w-\lambda) \frac{K_{\lambda}(w)}{K_{\lambda}(\lambda)}
$$

and further, using (16),

$$
\begin{aligned}
T_{f} K_{\lambda}(z) & =\int_{\mathbf{C}} \overline{K_{z}(w)} f(w) K_{\lambda}(w) d \mu_{\alpha}(w) \\
& =\int_{\mathbf{C}} K_{\lambda}(z) \overline{K_{z-\lambda}(w-\lambda)} \frac{\overline{K_{\lambda}(w)}}{K_{\lambda}(\lambda)} f(w) K_{\lambda}(w) d \mu_{\alpha}(w) \\
& =K_{\lambda}(z) \int_{\mathbf{C}}\left|k_{\lambda}(w)\right|^{2} \overline{K_{z-\lambda}(w-\lambda)} f(w) d \mu_{\alpha}(w) \\
& =K_{\lambda}(z) \int_{\mathbf{C}} f\left(t_{\lambda}(w)\right) \overline{K_{z-\lambda}(w)} d \mu_{\alpha}(w)=K_{\lambda}(z) P\left(f \circ t_{\lambda}\right)(z-\lambda)
\end{aligned}
$$

and $T_{f} k_{\lambda}(z)=k_{\lambda}(z) P\left(f \circ t_{\lambda}\right)(z-\lambda)$. Therefore, for $f \in L^{\infty}$ and $\lambda \in \mathbf{C}$,

$$
T_{f}\left(K_{\lambda}\right)=K_{\lambda} P\left(f \circ t_{\lambda}\right) \circ \tau_{\lambda}
$$

and

$$
H_{f}\left(K_{\lambda}\right)=K_{\lambda}\left(f-P\left(f \circ t_{\lambda}\right) \circ \tau_{\lambda}\right)
$$

Similarly,

$$
T_{f} k_{\lambda}=k_{\lambda} P\left(f \circ \varphi_{\lambda}\right) \circ \varphi_{\lambda},
$$

which will be used later together with the pointwise estimate

$$
\left|P\left(f \circ \varphi_{\lambda}\right)(z)\right| \leq \text { const } e^{\frac{\alpha}{4}|z|^{2}}
$$

for $z \in \mathbf{C}$ (see Lemma 6.25 of [19]).

\section{Boundedness and compactness}

In this section we study the fundamental properties of Toeplitz and Hankel operators that are needed in the study of their Fredholm theory. In particular, we recall some results on their boundedness and compactness in $F_{\alpha}^{2}$, and then generalize some of the results to the other Fock spaces $F_{\alpha}^{p}$.

First, it is clear that if the symbol $f$ is in $L^{\infty}$, then both the Toeplitz operator $T_{a}$ and the Hankel operator $H_{a}$ are bounded on $F_{\alpha}^{p}$, and it is easy to see that

$$
\left\|T_{f}\right\| \leq\|P\|\|f\|_{\infty} \text { and }\left\|H_{f}\right\| \leq\|P\|\|f\|_{\infty} .
$$

For unbounded symbols and measures as symbols, see [15]. 
The following result of [2] provides a complete characterization of compact Toeplitz operators on $F_{\alpha}^{p}$ with bounded symbols. The characterization is given in terms of the Berezin transform $\tilde{f}$ of the symbol $f$ defined by

$$
\begin{aligned}
\tilde{f}(z) & =\int_{\mathbf{C}}\left|k_{z}(w)\right|^{2} f(w) d \mu_{\alpha}(w)=\frac{\alpha}{\pi} \int_{\mathbf{C}} f(w) e^{-\alpha|z-w|^{2}} d A(w) \\
& =\int_{\mathbf{C}} f(z \pm w) d \mu_{\alpha}(w) .
\end{aligned}
$$

For Toeplitz operators on the Fock space $F^{2}$ with symbols in $B M O^{1}$, see [6], and for operators on unweighted Fock spaces $F^{p}$ with $0<p<\infty$, see [9].

Theorem 1. Let $f \in L^{\infty}, 1<p<\infty$ and $\alpha>0$. Then $T_{f}$ is compact on $F_{\alpha}^{p}$ if and only if $\tilde{f}$ vanishes at infinity.

Proof. See [2].

The following alternate characterization for Toeplitz operators on $F^{2}$ was proved in [17]. We generalize it to the other values of $p$.

Theorem 2. Let $f \in L^{\infty}, 1<p<\infty$ and $\alpha>0$. Then the following conditions are equivalent:

(a) $T_{f}$ is compact on $F_{\alpha}^{p}$;

(b) $\left\|P\left(f \circ t_{\lambda}\right)\right\|_{p, \alpha} \rightarrow 0$ as $|\lambda| \rightarrow \infty$.

Proof. Suppose that $T_{f}$ is compact on $F_{\alpha}^{p}$. If $g$ is a polynomial, then

$$
\left(g, k_{\lambda}\right)=\int_{\mathbf{C}} g(z) \overline{k_{\lambda}(z)} d \lambda_{\alpha}(z)=K_{\lambda}(\lambda)^{-1 / 2} \int_{\mathbf{C}} g(z) \overline{K_{\lambda}(z)} d \lambda_{\alpha}(z)=\frac{g(\lambda)}{e^{\frac{\alpha}{2}|\lambda|^{2}}} \rightarrow 0
$$

as $|\lambda| \rightarrow \infty$. Therefore, since the polynomials are dense in $F_{\alpha}^{p}$ (see [19, Proposition 2.9]), it follows that $k_{\lambda} \rightarrow 0$ weakly as $|\lambda| \rightarrow \infty$, and hence $\left\|T_{f} k_{\lambda}\right\|_{p} \rightarrow 0$ as $|\lambda| \rightarrow \infty$. Using (17), we get

$$
\begin{aligned}
\left\|T_{f} k_{\lambda}\right\|_{p, \alpha}^{p} & =\int_{\mathbf{C}}\left|k_{\alpha}(z, \lambda)\right|^{p}\left|P\left(f \circ t_{\lambda}\right)\left(\tau_{\lambda}(z)\right)\right|^{p} d \mu_{\alpha p / 2}(z) \\
& =\int_{\mathbf{C}}\left|P\left(f \circ t_{\lambda}\right)(w)\right|^{p} d \lambda_{\alpha p / 2}(w)=\left\|P\left(f \circ t_{\lambda}\right)\right\|_{p, \alpha}^{p},
\end{aligned}
$$

where we also used a change of variables according to (16). Thus, $\left\|P\left(f \circ t_{\lambda}\right)\right\|_{p, \alpha} \rightarrow 0$ as $|\lambda| \rightarrow \infty$.

Suppose that (b) holds. Then, using (17), we have

$$
|\tilde{f}(\lambda)|=\left|\left\langle T_{f} k_{\lambda}, k_{\lambda}\right\rangle_{\alpha}\right| \leq\left\|T_{f} k_{\lambda}\right\|_{p, \alpha}\left\|k_{\lambda}\right\|_{p^{\prime}, \alpha}=\left\|P\left(f \circ t_{\lambda}\right)\right\|_{p, \alpha} \rightarrow 0,
$$

where $1 / p+1 / p^{\prime}=1$. By the previous theorem, $T_{f}$ is compact on $F_{\alpha}^{p}$.

A similar result holds for Hankel operators as seen in the following theorem, which was proved in [17] for Hankel operators from $F^{2}$ to $L^{2}$ and in [19] for Hankel operators on $F_{\alpha}^{2}$ to $L_{\alpha}^{2}$.

Theorem 3. Let $f \in L^{\infty}$ and $\alpha>0$. Then the following conditions are equivalent:

(a) $H_{f}: F_{\alpha}^{2} \rightarrow L_{\alpha}^{2}$ is compact;

(b) $\left\|f \circ t_{\lambda}-P\left(f \circ t_{\lambda}\right)\right\|_{2, \alpha} \rightarrow 0$ as $|\lambda| \rightarrow \infty$. 
As mentioned in [17], when Hankel operators are acting on $F^{2}$, the equivalence of (a) and (b) in the following theorem was obtained in [3], while condition (d) was proved in [4]. An elementary proof of the theorem was given in [17].

Theorem 4. Let $f \in L^{\infty}$ and $\alpha>0$. Then the following statements are equivalent:

(a) $H_{f}: F_{\alpha}^{2} \rightarrow L_{\alpha}^{2}$ is compact;

(b) $H_{\bar{f}}: F_{\alpha}^{2} \rightarrow L_{\alpha}^{2}$ is compact;

(c) $\left\|f \circ t_{\lambda}-\tilde{f}(\lambda)\right\|_{2, \alpha} \rightarrow 0$ as $\lambda \rightarrow \infty$;

(d) $\widetilde{|f|^{2}}(\lambda)-|\tilde{f}(\lambda)|^{2} \rightarrow 0$ as $\lambda \rightarrow \infty$.

One remarkable difference between Fock, Bergman and Hardy spaces is that the equivalence of (a) and (b) in the preceding theorem is only true in Fock spaces. This is often explained by the lack of bounded analytic functions in $F_{\alpha}^{2}$.

We will study the Fredholm properties of Toeplitz operators with bounded symbols that satisfy an additional condition on their (mean) oscillation at infinity. These types of conditions are intimately related to the boundedness and compactness properties of Hankel operators. For $r>0$, define the local oscillation of a continuous function $f: \mathbf{C} \rightarrow \mathbf{C}$ at $z \in \mathbf{C}$ by

$$
\omega_{r}(f)(z)=\sup _{w \in B(z, r)}|f(z)-f(w)| .
$$

We say that $f$ is of bounded oscillation and write $f \in B O_{r}$ if $f$ is continuous and $\omega_{r}(f)$ is bounded on $\mathbf{C}$.

We define the mean average $\hat{f}_{r}(z)$ of a locally integrable function $f$ over $B(z, r)$ by

$$
\hat{f}_{r}(z)=\frac{1}{\pi r^{2}} \int_{D(z, r)} f(w) d A(w),
$$

and say that $f$ is of bounded average and write $f \in B A_{r}^{p}$ if $\widehat{|f|_{r}^{p}}(z)$ is bounded on $\mathbf{C}$; that is, if

$$
\sup _{z \in \mathbf{C}} \frac{1}{\pi r^{2}} \int_{B(z, r)}|f(w)|^{p} d A(w)<\infty .
$$

We say that $f$ is of vanishing oscillation and write $f \in V O_{r}$ if $f \in B O_{r}$ and

$$
\lim _{z \rightarrow \infty} \omega_{r}(f)=0 \text {. }
$$

Similarly, we say that $f$ is of vanishing average and write $f \in V A_{r}^{p}$ if

$$
\lim _{z \rightarrow \infty} \frac{1}{\pi r^{2}} \int_{B(z, r)}|f(w)|^{p} d A(w)=0 .
$$

Finally, we say that a locally integrable function $f$ is of bounded mean oscillation and write $f \in B M O_{r}^{p}$ if

$$
\sup _{z \in \mathbf{C}} \int_{B(z, r)}\left|f(w)-\hat{f}_{r}(z)\right|^{p} d A(w)<\infty .
$$

Similarly, we say that $f \in B M O_{r}^{p}$ is of vanishing mean oscillation and write $f \in$ $V M O_{r}^{p}$ if

$$
\lim _{z \rightarrow \infty} \int_{B(z, r)}\left|f(w)-\hat{f}_{r}(z)\right|^{p} d A(w)=0
$$


The spaces $B O_{r}, B A_{r}^{p}, V O_{r}, V A_{r}^{p}, B M O_{r}^{p}, V M O_{r}^{p}$ are all independent of $r$ and we often omit the subscript. However, they do depend on the value $p$, unlike in the setting of Hardy spaces; in particular, for $1 \leq p<q$, we have $B M O^{q} \subset B M O^{p}$ and $V M O^{q} \subset V M O^{p}$ with proper inclusions.

It is also well know that for $p \geq 1$,

$$
B M O^{p}=B O+B A^{p} \text { and } V M O^{p}=V O+V A^{p}
$$

and each $f$ in $B M O^{p}$ can be decomposed as the sums

$$
f=\tilde{f}+(f-\tilde{f}) \quad \text { or } \quad f=\hat{f}+(f-\hat{f}),
$$

where $\tilde{f}, \hat{f} \in B O$ and $f-\tilde{f}, f-\hat{f} \in B A^{p}$. Analogous decompositions hold for functions in $V M O^{p}$ (see, e.g., [13] or [19]).

Proposition 5. For $1<p<\infty$,

$$
L^{\infty} \cap B M O^{p}=L^{\infty} \cap B M O^{1} \text { and } L^{\infty} \cap V M O^{p}=L^{\infty} \cap V M O^{1} .
$$

Proof. Let $f \in L^{\infty} \cap V M O^{1}$. Then

$$
\left|f(w)-\hat{f}_{r}(z)\right| \leq 2\|f\|_{\infty}
$$

for all $w, z \in \mathbf{C}$. Thus,

$$
\int_{B(z, r)}\left|f(w)-\hat{f}_{r}(z)\right|^{p} d A(w) \leq\left(2\|f\|_{\infty}\right)^{p-1} \int_{B(z, r)}\left|f(w)-\hat{f}_{r}(z)\right| d A(w) \rightarrow 0
$$

as $|z| \rightarrow \infty$, which implies that $f \in V M O^{p}$. The other case is analogous.

The following theorem characterizes simultaneous boundedness (and compactness) of Hankel operators $H_{a}$ and $H_{\bar{a}}$; see [13]. It was first proved for Hankel operators on $F^{2}$ in [1]; see also [4]. Further generalizations to other Fock spaces $F_{\phi}^{p}$ can be found in [10]. The requirement that $f K_{w} \in L_{\alpha}^{p}$ for all $w \in \mathbf{C}$ in the theorem is natural because linear combinations of the kernel functions $K_{w}$ form a dense subset of $F_{\alpha}^{p}$. Observe that this condition is satisfied for all $p \in(1, \infty)$ and $\alpha>0$ if we have $f \in L^{\infty}$.

Theorem 6. Let $1<p<\infty, \alpha>0$ and suppose that $f K_{w} \in L_{\alpha}^{p}$. Then

(a) $H_{f}$ and $H_{\bar{f}}$ are both bounded $F_{\alpha}^{p} \rightarrow L_{\alpha}^{p}$ if and only if $f \in B M O^{p}$;

(b) $H_{f}$ and $H_{\bar{f}}$ are both compact $F_{\alpha}^{p} \rightarrow L_{\alpha}^{p}$ if and only if $f \in V M O^{p}$.

Proposition 7. For $\alpha>0$,

$$
\left\{f \in L^{\infty}: H_{f} \in K\left(F_{\alpha}^{2}, L_{\alpha}^{2}\right)\right\}=L^{\infty} \cap V M O^{2} .
$$

Proof. Apply Theorems 4 and 6.

Proposition 8. The space $L^{\infty} \cap V M O^{1}$ equipped with $\|\cdot\|_{\infty}$ is a Banach algebra.

Proof. Let $f, g \in L^{\infty} \cap V M O^{1}=L^{\infty} \cap V M O^{2}$ (see Proposition 5). Then

$$
H_{f g}=Q M_{f g}=Q M_{f}(P+Q) M_{g}=Q M_{f} P M_{g}+Q M_{f} Q M_{g}
$$

is compact from $F_{\alpha}^{2}$ to $L_{\alpha}^{2}$, and $f g \in L^{\infty}$. Therefore, by the previous proposition, it follows that the function $f g$ is in $L^{\infty} \cap V M O^{1}$. 


\section{Fredholm theory}

In this section we state and prove our main results. We start with the definition of Fredholm operators. Let $T$ be a bounded linear operator on a Banach space $X$. We say that $T$ is a Fredholm operator on $X$ if

$$
d_{1}=\operatorname{dim} \operatorname{ker} T<\infty \quad \text { and } \quad d_{2}=\operatorname{dim}(X / T(X)),
$$

in which case we define the index of $T$ to be ind $T=d_{1}-d_{2}$. The set of all Fredholm operators on $X$ is denoted by $\Phi(X)$. The definition of Fredholm operators immediately gives rise to the concept of the essential spectrum $\sigma_{\text {ess }}(T)$ of a bounded operator $T$ on $X$ defined by

$$
\sigma_{\mathrm{ess}}(T)=\{\lambda \in \mathbf{C}: T-\lambda I \notin \Phi(X)\},
$$

where $I$ is the identity operator on $X$. Clearly $\sigma_{\text {ess }}(T) \subset \sigma(T)$. A useful characterization of Fredholm operators can be given in terms of invertibility in the Calkin algebra; that is, $T$ is Fredholm if and only if $T+K(X)$ is invertible in $B(X) / K(X)$. We also recall that the index function is stable under small perturbations.

The following "approximation" result plays an important role in our analysis; cf. [3]. We say that a function $f: \mathbf{C} \rightarrow \mathbf{C}$ is bounded away from zero if there is a $R>0$ and an $m>0$ such that

$$
|f(z)| \geq m \text { for }|z| \geq R
$$

Note also that in what follows, we only need the case of functions of vanishing oscillation. However, the other case may turn out to be of interest in further considerations of the essential spectra of Toeplitz operators.

Proposition 9. Let $f: \mathbf{C} \rightarrow \mathbf{C}$ be a continuous function in $A$, where $A=$ $L^{\infty} \cap V O$ or $A=L^{\infty} \cap V M O^{1}$. Then $f$ is bounded away from zero on $\mathbf{C} \backslash B(0, R)$ for some $R>0$ if and only if there is a continuous function $g \in A$ such that $g(z) f(z) \rightarrow 1$ as $z \rightarrow \infty$.

Proof. Suppose first that such a function $g$ exists. Then $g f-1$ vanishes at infinity. If $f$ is not bounded away from zero for any $R>0$, then there are $z_{k} \in \mathbf{C}$ such that $\left|z_{k}\right| \rightarrow \infty$ and $\left|f\left(z_{k}\right)\right| \rightarrow 0$, and so $\left|1+g\left(z_{k}\right) f\left(z_{k}\right)-1\right| \leq\|g\|_{\infty}\left|f\left(z_{k}\right)\right| \rightarrow 0$, which is a contradiction.

Conversely, suppose that there is an $m>0$ such that $|f(z)| \geq m$ for $|z| \geq R$. Denote by $\kappa$ the winding number of $f$ along the circle $C_{R}=\{|z|=R\}$. Consider the function $h$ defined by

$$
h(z)=f(z)(\bar{z} /|z|)^{\kappa}
$$

for $|z| \geq R$. Since wind $h=0$ along $\partial B(0, R)$, the function extends to a bounded continuous function on the whole complex plane which is bounded away from zero on $\mathbf{C}$.

We show that $h \in A$. Suppose first that $A=L^{\infty} \cap V O$. For $|z-w| \leq 1$,

$$
\left|(\bar{z} /|z|)^{\kappa}-(\bar{w} /|w|)^{\kappa}\right|=O(1 /|z|)
$$

as $z \rightarrow \infty$. Therefore, since $f \in L^{\infty} \cap V O$ and

$$
|h(z)-h(w)|=|f(z)|\left|(\bar{z} /|z|)^{\kappa}-(\bar{w} /|w|)^{\kappa}\right|+|f(z)-f(w)|,
$$


it follows that $h \in L^{\infty} \cap V O$. Suppose then that $A=L^{\infty} \cap V M O^{1}$. For $w \in D(z, 1)$,

$$
\begin{aligned}
h(w)-\hat{h}(z)= & \mid h(w)-\frac{1}{\pi} \int_{D(z, 1)} h(u) d A(u) \\
=\frac{1}{\pi} & \int_{D(z, 1)}\left(h(w)-f(w)(\bar{z} /|z|)^{\kappa}\right. \\
& \left.\quad+f(w)(\bar{z} /|z|)^{\kappa}-f(u)(\bar{z} /|z|)^{\kappa}+f(u)(\bar{z} /|z|)^{\kappa}-h(u)\right) d A(u) \\
=\frac{1}{\pi} & \int_{D(z, 1)}\left(f(w)\left((\bar{w} /|w|)^{\kappa}-(\bar{z} /|z|)^{\kappa}\right)\right. \\
& \left.\quad+(f(w)-f(u))(\bar{z} /|z|)^{\kappa}+f(u)\left((\bar{z} /|z|)^{\kappa}-(\bar{u} /|u|)^{\kappa}\right)\right) d A(u) .
\end{aligned}
$$

Therefore,

$$
\frac{1}{\pi} \int_{D(z, 1)}|h(w)-\hat{h}(z)| d A(z)=O(1 / z)+\frac{1}{\pi} \int_{D(z, 1)}|f(w)-\hat{f}(z)| d A(z)+O(1 / z),
$$

which shows that $h \in V M O^{1}$. Finally, we define a function $F$ on $\mathbf{C}$ by setting

$$
F(z)= \begin{cases}(\bar{z} /|z|)^{\kappa}, & |z| \geq R, \\ (\bar{z}) / R)^{\kappa}, & |z|<R\end{cases}
$$

and let $g=F / h$. Then $g \in A$ and $g(z) f(z)=1$ for $|z| \geq R$.

In 1987 Berger and Coburn [3] characterized Fredholm Toeplitz operators on the standard Fock space $F^{2}$ for bounded symbols of vanishing oscillation at infinity. Their approach was based on heavy machinery employing Hilbert space and $C^{*}$ algebra techniques, making use of the properties of the Weyl operator, of the Berezin symbol, and of the Heisenberg group. These are by no means ideal techniques to be used in the Banach space context. In 1992 Stroethoff [17] gave a more elementary proof of Berger and Coburn's result using ideas which are more applicable in the setting of the standard weighted Fock spaces $F_{\alpha}^{p}$. Before we state our main result we need the following results, which allows us to use the Berezin transform in our analysis.

Lemma 10. If $f \in V O \cap L^{\infty}$, then $f(z)-\tilde{f}(z) \rightarrow 0$ as $|z| \rightarrow \infty$.

Proof. Let $\epsilon>0$. By direct computation, there is an $M>0$ such that

$$
\begin{aligned}
|f(z)-\tilde{f}(z)| \leq & \frac{\alpha}{\pi} \int_{\mathbf{C}}|f(z)-f(w)| e^{-\alpha|z-w|^{2}} d A(w) \\
= & \frac{\alpha}{\pi} \int_{\mathbf{C}}|f(z)-f(z+w)| e^{-\alpha|w|^{2}} d A(w) \\
\leq & \frac{\alpha}{\pi} 2\|f\|_{\infty} \int_{|w| \geq M} e^{-\alpha|w|^{2}} d A(w) \\
& +\frac{\alpha}{\pi} \int_{|w|<M}|f(z)-f(z+w)| e^{-\alpha|w|^{2}} d A(w) \\
\leq & \epsilon+\frac{\alpha}{\pi} \int_{|w|<M}|f(z)-f(z+w)| e^{-\alpha|w|^{2}} d A(w) .
\end{aligned}
$$

Since $f \in V O$, the last integral goes to zero as $|z| \rightarrow \infty$, and the proof is complete. 
Corollary 11. Let $f \in V O$. Then $\tilde{f}(z) \rightarrow 0$ as $|z| \rightarrow \infty$ if and only if $f(z) \rightarrow 0$ as $|z| \rightarrow \infty$.

Proposition 12. Let $f \in L^{\infty} \cap V M O^{1}, 1<p<\infty$, and $\alpha>0$. Then $T_{f-\tilde{f}}$ is compact on $F_{\alpha}^{p}$.

Proof. This follows from Theorem 1.

We can now characterize Fredholmness of Toeplitz operators in terms of their symbols.

Theorem 13. Let $\alpha>0,1<p<\infty$, and suppose that $f$ is in $L^{\infty} \cap V O$. Then the Toeplitz operator $T_{f}$ is Fredholm on $F_{\alpha}^{p}$ if and only the symbol $f$ is bounded away from zero; see (28).

Proof. Suppose that $f$ is bounded away from zero. We use Proposition 9 to construct a regularizer. Indeed, there is a function $g$ in $L^{\infty} \cap V O$ such that $f(z) g(z)-$ $1 \rightarrow 0$ as $z \rightarrow \infty$. By Theorem $6, H_{f}$ and $H_{g}$ are compact, and by Theorem $1, T_{f g-1}$ is compact. Therefore,

$$
\begin{aligned}
T_{f} T_{g} & =P M_{f} P M_{g}=P M_{f}(I-Q) M_{g}=T_{f g}-P M_{f} Q M_{g} \\
& =T_{f g}-P M_{f} H_{g}=I+T_{f g-1}-P M_{f} H_{g}=I+K_{1}
\end{aligned}
$$

for some compact operator $K_{1}$. Similarly, $T_{g} T_{f}=I+T_{f g-1}-P M_{g} H_{f}=I+K_{2}$ for $K_{2}$ compact. Thus, $T_{g}$ is a regularizer for $T_{f}$ and hence $T_{f}$ is Fredholm.

Conversely, suppose that there are $\lambda_{k} \in \mathbf{C}$ such that $f\left(\lambda_{k}\right) \rightarrow 0$ when $\left|\lambda_{k}\right| \rightarrow \infty$. By Lemma 11, $\tilde{f}\left(\lambda_{k}\right) \rightarrow 0$ as $k \rightarrow \infty$. By (19), we have

$$
\left\|T_{f} k_{\lambda_{k}}\right\|_{p, \alpha}^{p}=\left\|P_{\alpha}\left(f \circ t_{\lambda_{k}}\right)\right\|_{p, \alpha}^{p}=\mathrm{const} \int_{\mathbf{C}}\left|P\left(f \circ t_{\lambda_{k}}\right)(z)\right|^{p} d \mu_{\alpha p / 2}(z) .
$$

By Lemma 6.26 of [19], for $z \in \mathbf{C}, P\left(f \circ \varphi_{\lambda_{k}}\right)(z) \rightarrow 0$ as $k \rightarrow \infty$. Therefore, by (20),

$$
\left|k_{z}\left(\lambda_{k}-z\right) P\left(f \circ \varphi_{\lambda_{k}}\right)(z) e^{-\frac{1}{2}\left|\lambda_{k}-z\right|^{2}}\right|=e^{-\frac{\alpha}{2}|z|^{2}}\left|P\left(f \circ \varphi_{\lambda_{k}}\right)(z)\right| \leq \text { const } e^{-\frac{\alpha}{4}|z|^{2}},
$$

and so by the Lebesgue dominated convergence theorem,

$$
\begin{aligned}
\left\|T_{f} k_{\lambda_{k}}\right\|_{p, \alpha}^{p} & =\mathrm{const} \int_{\mathbf{C}}\left|k_{\lambda_{k}}(z) P\left(f \circ \varphi_{\lambda_{k}}\right)\left(\lambda_{k}-z\right) e^{\frac{\alpha}{2}|z|^{2}}\right|^{p} d A(z) \\
& =\int_{\mathbf{C}}\left|k_{z}\left(\lambda_{k}-z\right) P\left(f \circ \varphi_{\lambda_{k}}\right)(z) e^{-\frac{1}{2}\left|\lambda_{k}-z\right|^{2}}\right|^{p} d A(z) \rightarrow 0
\end{aligned}
$$

as $k \rightarrow \infty$. Thus, $T_{f}+K$ is not invertible in the Calkin algebra $L\left(F_{\alpha}^{p}\right) / K\left(F_{\alpha}^{p}\right)$, and hence $T_{f}$ is not Fredholm on $F_{\alpha}^{p}$.

Corollary 14. Let $f \in L^{\infty} \cap V O$. Then the essential spectrum of $T_{f}$ on $F_{\alpha}^{p}$ with $1<p<\infty$ and $\alpha>0$ is given by

$$
\sigma_{\text {ess }}\left(T_{f}\right)=\bigcap_{R>0} \operatorname{cl} f(\mathbf{C} \backslash R \mathbf{D}) .
$$

By using the result on the compactness of Toeplitz operators, we can consider a slightly larger class of symbols.

Corollary 15. Let $\alpha>0,1<p<\infty$, and suppose that $f$ is in $L^{\infty} \cap V M O^{1}$. Then the Toeplitz operator $T_{f}$ is Fredholm on $F_{\alpha}^{p}$ if and only if $\tilde{f}$ is bounded away from zero for some $R>0$; see (28). 
Proof. Write $f=\tilde{f}+(f-\tilde{f})$ as in (26). Then $\tilde{f} \in V O$ and $f-\tilde{f} \in V A^{1}$. Since $T_{f-\tilde{f}}$ is compact, the standard Fredholm theory implies that $T_{f}$ is Fredholm if and only if $T_{\tilde{f}}$ is Fredholm, which is equivalent to $\tilde{f}$ being bounded away from zero by the preceding theorem.

Corollary 16. Let $f \in L^{\infty} \cap V M O^{1}$. Then the essential spectrum of $T_{f}$ on $F_{\alpha}^{p}$ with $1<p<\infty$ and $\alpha>0$ is given by

$$
\sigma_{\mathrm{ess}}\left(T_{f}\right)=\bigcap_{R>0} \operatorname{cl} \tilde{f}(\mathbf{C} \backslash R \mathbf{D}) .
$$

Proof. This follows from Theorem 13 and the fact that $\sigma_{\text {ess }}\left(T_{f}\right)=\sigma_{\text {ess }}\left(T_{\tilde{f}}\right)$, which is easy to see using Proposition 12 and standard Fredholm theory.

The description of the essential spectrum of $T_{f}$ in terms of Berezin transform $\tilde{f}$ above comes useful because $\tilde{f}$ is continuous and hence the sets $\mathrm{cl} \tilde{f}(\mathbf{C} \backslash R \mathbf{D})$ are compact and connected, which immediately proves the following result.

Corollary 17. Let $f \in L^{\infty} \cap V M O^{1}, 1<p<\infty$, and $\alpha>0$. Then the essential spectrum $\sigma_{\text {ess }}\left(T_{f}\right)$ of $T_{f}$ on $F_{\alpha}^{p}$ is connected.

We reduce the computation of the index of $T_{f}$ to that of $T_{\bar{z} /|z|}$. To compute the index of the latter simple operator, we use the following two lemmas on weighted shift operators.

Lemma 18. Let $\alpha>0$ and $1<p<\infty$. The Toeplitz operator $T_{z /|z|}$ is a weighted shift operator on $F_{\alpha}^{p}$; that is, $T_{z /|z|} e_{k}=\alpha_{k} e_{k+1}$, where $e_{k}=\sqrt{\frac{\alpha^{k}}{k !}} z^{k}$ and $\alpha_{k} \neq 0$.

Proof. Set $g(z)=z /|z|$ for $z \in \mathbf{C}$. If $w=r e^{i \theta}$, then $d A(w)=r d r d \theta$ and

$$
\begin{aligned}
T_{g} e_{k}(z) & =P\left(g e_{k}\right)(z)=\frac{\alpha}{\pi} \int_{\mathbf{C}} e^{\alpha z \bar{w}-\alpha|w|^{2}}\left(e^{i \theta} e_{k}\right) d A(w) \\
& =\frac{\alpha}{\pi} \int_{\mathbf{C}} e^{\alpha z \bar{w}-\alpha|w|^{2}} e^{i \theta} \sqrt{\frac{\alpha^{k}}{k !}} z^{k} d A(w) \\
& =\frac{\alpha}{\pi} \sqrt{\frac{\alpha^{k}}{k !}} \int_{0}^{\infty} r^{k+1} e^{-\alpha r^{2}}\left[\int_{0}^{2 \pi} e^{\alpha z r e^{-i \theta}} e^{i \theta(k+1)} d \theta\right] d r \\
& =\frac{\alpha}{\pi} \sqrt{\frac{\alpha^{k}}{k !}} \int_{0}^{\infty} r^{k+1} e^{-\alpha r^{2}}\left[\int_{|\lambda|=1}(-i) e^{\alpha z r \lambda} \frac{d \lambda}{\lambda^{k+2}}\right] d r,
\end{aligned}
$$

where $e^{-i \theta}=\lambda$. By the residue theorem,

$$
\int_{|\lambda|=1} \frac{e^{\mu \lambda}}{\lambda^{k+2}} d \lambda=\frac{2 \pi i \mu^{k+1}}{(k+1) !}
$$

Therefore,

$$
T_{g} e_{k}(z)=2 \alpha \sqrt{\frac{\alpha^{k}}{k !}} \frac{1}{(k+1) !} \alpha^{k+1} z^{k+1} \int_{0}^{\infty} r^{2 k+2} e^{-\alpha r^{2}} d r=\alpha_{k} e_{k+1}(z),
$$

where

$$
\alpha_{k}=\frac{\alpha^{k} \sqrt{\alpha}}{k ! \sqrt{k+1}} \int_{0}^{\infty} r^{2 k+2} e^{-\alpha r^{2}} d r \neq 0
$$

for $k \in \mathbf{N}$. 
Next we compute the index of $T_{\bar{z} /|z|}$ using the properties of the diagonal operator.

Lemma 19. Let $\alpha>0$ and $1<p<\infty$. Then $\operatorname{ind} T_{\bar{z} /|z|}=1$.

Proof. Let $g \in F_{\alpha}^{p}$, then $g(z)=\sum_{k=0}^{\infty} \lambda_{k} e_{k}$. Using Lemma 18, we get

$$
T_{\frac{z}{|z|}}(g)(z)=\sum_{k=0}^{\infty} \lambda_{k} T_{\frac{z}{|z|}} e_{k}=\sum_{k=0}^{\infty} \lambda_{k} \alpha_{k} e_{k+1}, \alpha_{k} \neq 0 \quad \text { for all } k \geq 0 .
$$

Thus, $g \in \operatorname{ker} T_{\frac{z}{|z|}}$ if and only if $\sum_{k=0}^{\infty} \lambda_{k} \alpha_{k} e_{k+1}=0$. Hence, $g \in \operatorname{ker} T_{\frac{z}{|z|}}$ if and only if $\lambda_{k}=0$ for all $k \geq 0$. This implies that $\operatorname{ker} T_{\frac{z}{|z|}}=0$. Moreover, if $h(z)=a e_{0}$ then $h \notin$ Range $T_{\frac{z}{|z|}}$, this means that Codim $T_{\frac{z}{|z|}}=1$. Therefore, $T_{\frac{z}{|z|}}$ is Fredholm with index 1. This implies that $T_{\frac{z}{|z|}}^{*}=T_{\frac{z}{|z|}}$ is Fredholm with index -1 .

We can now prove the index formula for Toeplitz operators on $F_{\alpha}^{p}$ with continuous symbols of vanishing oscillation.

Theorem 20. Let $f \in L^{\infty} \cap V O, \alpha>0$, and $1<p<\infty$. If $f$ is bounded away from zero, then

$$
\text { ind } T_{f}=-\operatorname{wind}\left(\left.f\right|_{|z|=R}\right) \text {, }
$$

where $R$ is any positive number for which $|f(z)|>m>0$ for $|z| \geq R$ and wind $\left.f\right|_{\{|z|=R\}}$ is the winding of the curve $f(\{|z|=R\})$ around the origin.

Proof. By Theorem 13, $T_{f}$ is Fredholm. Suppose wind $\left(\left.f\right|_{|z|=R}\right)=n$. By Proposition 9 and its proof there is an $H$ in $L^{\infty}(\mathbf{C}) \cap V O$ such that $H$ is bounded away from zero on the whole complex plane and

$$
\lim _{z \rightarrow \infty}\left[(\bar{z} /|z|)^{n} f(z)-H(z)\right]=0 .
$$

It follows that $T_{(\bar{z} /|z|)^{n} f-H}$ is compact by Theorem 1 . Therefore, since $T_{H}$ is Fredholm (see Theorem 13), we have

$$
\text { ind }\left(T_{(\bar{z} /|z|)^{n}} T_{f}\right)=\operatorname{ind} T_{(\bar{z} /|z|)^{n} f}=\operatorname{ind} T_{H} .
$$

By the index theorem and Lemma 19,

$$
\text { ind } T_{f}=-n+\operatorname{ind} T_{H} \text {. }
$$

It remains to show that ind $T_{H}=0$. Define

$$
h_{t}=\frac{t}{|H|}+1-t
$$

for $t \in[0,1]$. Then each $T_{h_{t}}$ is Fredholm and

$$
\left\|T_{h_{t}}-T_{h_{s}}\right\| \leq \mathrm{const}|t-s|\left\||H|^{-1}-1\right\|,
$$

so

$$
\operatorname{ind} T_{1 /|H|}=\operatorname{ind} T_{h_{1}}=\operatorname{ind} T_{h_{0}}=0 \text {. }
$$

Therefore,

$$
\text { ind } T_{H /|H|}=\operatorname{ind} T_{H}+\operatorname{ind} T_{1 /|H|}=\operatorname{ind} T_{H},
$$

and hence we can assume that $|H|=1$ and write $H=e^{i \arg H}$. For any integer $m>0$, since $H$ is a continuous function of no winding,

$$
\exp (i(\arg H) / m) \in L^{\infty} \cap V O \text {. }
$$

Thus,

$$
\text { ind } T_{H}=\operatorname{ind} T_{\exp (i(\arg H) / m)}^{m}=m \operatorname{ind} T_{\exp (i(\arg H) / m)},
$$

which implies that ind $T_{H}=0$. 


\section{Matrix-valued symbols}

Let $N \in \mathbf{N}, 1 \leq p<\infty$, and $\alpha>0$. We define

$$
L_{\alpha, N}^{p}=\left\{\left(f_{1}, \ldots, f_{N}\right)^{T}: f_{k} \in L_{\alpha}^{p}\right\}
$$

and

$$
\|f\|_{\alpha, p, N}=\sum_{k=1}^{N}\left\|f_{k}\right\|_{\alpha}^{p}
$$

The vectorial Fock space

$$
F_{\alpha, N}^{p}=\left\{\left(f_{1}, \ldots, f_{N}\right)^{T}: f_{k} \in F_{\alpha}^{p}\right\}
$$

is a closed subspace of $L_{\alpha, N}^{p}$.

Given $a=\left(a_{j k}\right)_{1 \leq j, k \leq N}$, where $a_{j k} \in L^{\infty}$, we define the block Toeplitz operator $T_{a}$ on $F_{\alpha, N}^{p}$ by

$$
\begin{aligned}
& T_{a} f=P(a f)=P\left(\begin{array}{ccccccc}
a_{11} f_{1} & + & a_{12} f_{2} & + & \ldots & + & a_{1 N} f_{N} \\
\vdots & & \vdots & & \vdots & & \vdots \\
a_{N 1} f_{1} & + & a_{N 2} f_{2} & + & \ldots & + & a_{N N} f_{N}
\end{array}\right) \\
& =\left(\begin{array}{ccccccc}
P\left(a_{11} f_{1}\right) & + & P\left(a_{12} f_{2}\right) & + & \ldots & + & P\left(a_{1 N} f_{N}\right) \\
\vdots & & \vdots & & \vdots & & \vdots \\
P\left(a_{N 1} f_{1}\right) & + & P\left(a_{N 2} f_{2}\right) & + & \ldots & + & P\left(a_{N N} f_{N}\right)
\end{array}\right) \\
& =\left(\sum_{k=1}^{N} T_{a_{j k}} f_{k}\right)_{1 \leq j \leq N}^{T}=\left(\begin{array}{cccc}
T_{a_{11}} & T_{a_{12}} & \cdots & T_{a_{1 N}} \\
\vdots & \vdots & & \vdots \\
T_{a_{N 1}} & T_{a_{N 2}} & \cdots & T_{a_{N N}}
\end{array}\right)\left(\begin{array}{c}
f_{1} \\
\vdots \\
f_{N}
\end{array}\right)
\end{aligned}
$$

for $f \in F_{\alpha, N}^{p}$. It is not difficult to see that $T_{a}$ is bounded on $F_{\alpha, N}^{p}$.

Lemma 21. There is a compact operator $K$ such that $\operatorname{det} T_{a}=T_{\operatorname{det} a}+K$.

Proof. For an $N \times N$ matrix $A$, we have

$$
\operatorname{det} A=\sum_{\sigma \in S_{N}} \operatorname{sgn}(\sigma) \prod_{j=1}^{N} A_{j \sigma(j)},
$$

where $S_{N}$ is the group of $N$-permutations and $\operatorname{sgn}(\sigma)$ is the sign of each permutation. If $f, g \in L^{\infty} \cap V M O^{1}$, then

$$
T_{f} T_{g}=T_{f g}-P M_{f} H_{g}=T_{f g}+K
$$

for some compact operator $K$ since $H_{g}$ is compact by Theorem 6 . Therefore, there is a compact operator $K$ such that

$$
\begin{aligned}
\operatorname{det} T_{a} f & =\sum_{\sigma \in S_{N}} \operatorname{sgn}(\sigma) T a_{1 \sigma(1)} \cdots T_{N \sigma(N)}=\sum_{\sigma \in S_{N}} \operatorname{sgn}(\sigma) T_{a_{1 \sigma(1)} \cdots a_{N \sigma(N)}} f+K f \\
& =P\left(\sum_{\sigma \in S_{N}} \operatorname{sgn}(\sigma)\left(a_{1 \sigma(1) \cdots a_{N \sigma(N)}}\right) f\right)+K f=P((\operatorname{det} a) f)+K f \\
& =T_{\operatorname{det} a} f+K f .
\end{aligned}
$$

Theorem 22. Let $1<p<\infty$ and $\alpha>0$. If $a \in\left(L^{\infty} \cap V M O^{1}\right)_{N \times N}$ for some $N \in \mathbf{N}$. Then $T_{a}$ is Fredholm on $F_{\alpha, N}^{p}$ if and only if $\widetilde{\operatorname{det} a}$ is bounded away from zero. If $a \in\left(L^{\infty} \cap V O\right)_{N \times N}$ for some $N \in \mathbf{N}$. Then $T_{a}$ is Fredholm on $F_{\alpha, N}^{p}$ if and only if $\operatorname{det} a$ is bounded away from zero 
Proof. In the first case, since the entries of $T_{a}$ commute pairwise modulo compact operators, Theorem 1.14 of [5] implies that $T_{a}$ is Fredholm if and only if $\operatorname{det} T_{a}$ is Fredholm. By the previous lemma and Corollary 15, $T_{a}$ is Fredholm if and only if $\widetilde{\operatorname{det} a}$ is bounded away from zero. The other case is analogous.

We conjecture that if $a \in\left(L^{\infty} \cap V O\right)_{N \times N}$ and if $\operatorname{det} a$ is bounded away from zero for some $R>0$, then

$$
\operatorname{ind} T_{a}=\operatorname{ind} T_{\operatorname{det} a}=- \text { wind }\left(\left.(\operatorname{det} a)\right|_{\{|z|=R\}}\right) \text {. }
$$

Indeed, this true in some particular cases; e.g. when the entries of $T_{a}$ commute modulo trace class operators, which happens if and only if

$$
\int_{\mathbf{C}}\left(\widetilde{|f|^{2}}(z)-|\tilde{f}(z)|^{2}\right)^{1 / 2} d A(z)<\infty
$$

(see Theorem 8.26 of [19]). Another particular case in which the index formula (33) holds is provided by Theorem 7.6 of [8]. Analogous index formulas hold for Toeplitz operators on Hardy and Bergman spaces.

\section{References}

[1] BAuer, W.: Mean oscillation and Hankel operators on the Segal-Bargmann space. - Integral Equations Operator Theory 52:1, 2005, 1-15.

[2] Bauer, W., and J. Isralowitz: Compactness characterization of operators in the Toeplitz algebra of the Fock space $F_{\alpha}^{p}$. - J. Funct. Anal. 263:5, 2012, 1323-1355.

[3] Berger, C. A., and L. A. Coburn: Toeplitz operators on the Segal-Bargmann space. - Trans. Amer. Math. Soc. 301:2, 1987, 813-829.

[4] Berger, C. A., L. A. Coburn, and K. H. Zhu: Toeplitz operators and function theory in n-dimensions. - In: Pseudodifferential operators (Oberwolfach, 1986), Lecture Notes in Math. 1256, Springer, Berlin, 1987, 28-35.

[5] Böttcher, A., and B. Silbermann: Analysis of Toeplitz operators. - Springer Monogr. Math., Springer-Verlag, Berlin, second edition, 2006.

[6] Coburn, L. A., J. Isralowitz, and B. Li: Toeplitz operators with BMO symbols on the Segal-Bargmann space. - Trans. Amer. Math. Soc. 363:6, 2011, 3015-3030.

[7] Fulsche, R., and R. Hagger: Fredholmness of Toeplitz operators on the Fock space. Complex Anal. Oper. Theory (to appear).

[8] Gohberg, I., and S. Goldberg, and M. A. KaAshoek: Classes of linear operators. Vol. I. - Oper. Theory Adv. Appl. 49, Birkhäuser Verlag, Basel, 1990.

[9] Hu, Z., and X. Lv: Toeplitz operators on Fock spaces $F^{p}(\varphi)$. - Integral Equations Operator Theory 80:1, 2014, 33-59.

[10] Hu, Z., and X. Lv: Hankel operators on weighted Fock spaces. - Sci. China Math. 46:2, 2016, 141-156 (in Chinese).

[11] IsRalowitz, J., J. Virtanen, and L. Wolf: Schatten class Toeplitz operators on generalized Fock spaces. - J. Math. Anal. Appl. 421:1, 2015, 329-337.

[12] Isralowitz, J., and K. Zhu: Toeplitz operators on the Fock space. - Integral Equations Operator Theory 66:4, 2010, 593-611.

[13] Perälä, A., A. Schuster, and J. A. Virtanen: Hankel operators on Fock spaces. - In: Concrete operators, spectral theory, operators in harmonic analysis and approximation, Oper. Theory Adv. Appl. 236, Birkhäuser/Springer, Basel, 2014, 377-390.

[14] Perälä, A., J. TAskinen, and J. Virtanen: Toeplitz operators with distributional symbols on Fock spaces. - Funct. Approx. Comment. Math. 44:2, 2011, 203-213. 
[15] Schuster, A.P., and D. Varolin: Toeplitz operators and Carleson measures on generalized Bargmann-Fock spaces. - Integral Equations Operator Theory 72:3, 2012, 363-392.

[16] Seip, K., and E. H. Youssfi: Hankel operators on Fock spaces and related Bergman kernel estimates. - J. Geom. Anal. 23:1, 2013, 170-201.

[17] Stroethoff, K.: Hankel and Toeplitz operators on the Fock space. - Michigan Math. J. 39:1, 1992, 3-16.

[18] Vasilevski, N. L.: Commutative algebras of Toeplitz operators on the Bergman space. - Oper. Theory Adv. Appl. 185, Birkhäuser Verlag, Basel, 2008.

[19] ZHU, K.: Analysis on Fock spaces. - Graduate Texts in Math. 263, Springer, New York, 2012.

Received 6 December 2017 • Accepted 1 February 2018 